\title{
Managing distributed trust relationships for multi-modal authentication
}

\author{
Tim Van hamme, Davy Preuveneers, Wouter Joosen \\ \{tim.vanhamme, davy.preuveneers, wouter.joosen\}@cs.kuleuven.be \\ imec-DistriNet-KU Leuven \\ Celestijnenlaan 200A, B-3001 Heverlee, Belgium
}

\begin{abstract}
Multi-modal active authentication schemes fuse decisions of multiple behavioral biometrics (behaviometrics) to reduce identity verification errors. The challenge that we address in this work is the security risk caused by these decision fusion schemes making invalid assumptions, such as a fixed probability of (in)correct recognition and a temporal congruence of behaviometrics. To mitigate this risk, this paper presents a formal trust model that drives the behaviometric selection and composition. Our trust model adopts a hybrid approach combining policy and reputation based knowledge representation techniques. Our model and framework (1) externalizes trust knowledge from the authentication logic to achieve loosely coupled trust management, and (2) formalizes this knowledge in description logic to reason upon and broker complex distributed trust relationships to make risk-adaptive decisions for multi-modal authentication. The evaluation of our proof-of-concept illustrates an acceptable performance overhead while lifting the burden of manual trust and behaviometric management for multi-modal authentication.
\end{abstract}

Keywords: authentication, behavioral biometrics, reputation, trust model, ontology

\section{Introduction}

Due to the rapid evolution of cloud computing, Big Data, and the Internet of Things, enterprises rely more and more on context information about the user to improve the delivery, the user convenience and the security of their business applications and services. In multi-modal continuous authentication [1, 2], various behaviometrics of a subject are being collected in the background by distributed sensors or other information sources in order to jointly and continuously verify in a user-friendly manner the identity of the subject by establishing and recognizing unique behavioral patterns. The contribution of each behaviometric is usually weighed with a variant of Chair and Varshney's optimal decision fusion rule [3]. While such decision fusion schemes help to reduce the equal error rate (EER) of multi-modal authentication systems, they make assumptions that may jeopardize the trustworthiness of the authentication. For example, (1) decision fusion scheme implementations often mistakenly assume a static probability of correct identification and false alarms for each individual behaviometric; (2) they expect temporal congruence of the different behaviometric data streams; and (3) they ignore security threats of behaviometric sensors (e.g. sensors being temporarily disabled or compromised, and the fact that some behavior data is easily observed in public by adversaries that can use it to spoof a victim's identity).

In the face of risk and uncertainty, behaviometric authentication implementations must be able to understand and reason upon the trustworthiness of the sensors and the information they depend upon. Existing risk and trust models $[4,5,6]$ proposed in the literature over the past decades, are inadequate due to contextual dependencies and the dynamicity of behaviometrics - i.e. (mis)identification classification probabilities evolving over time - that have an impact on multi-modal continuous authentication. To address this challenge, we propose a trust model and framework that liberate the security architect and the end-user from manually selecting and composing behaviometrics by managing and reasoning upon complex trust relationships for all collaborating systems, sensors and applications for continuous risk-adaptive authentication.

This paper presents a formal representation of a trust model and supporting management framework that enables distributed applications to infer and broker trust relationships. Mainly driven by the requirements of our use case on continuous multi-modal authentication to establish trust in behaviometrics, our model adopts a hybrid approach combining policy and reputation based knowledge representation techniques. The contributions of our trust model and supporting framework are as follows:

1. It externalizes trust knowledge from the authentication logic to augment the core risk model with applicationspecific trust concepts while minimizing the impact on the authentication logic.

2. It formalizes risk, trust and context concepts in description logic to automate context-aware inference and brokerage of trust relationships.

3. The trust management framework has an acceptable low computational overhead to infer and broker these relationships.

While we will demonstrate and evaluate the feasibility, applicability and performance of our hybrid trust model with our authentication use case, its design should make it suitable for 


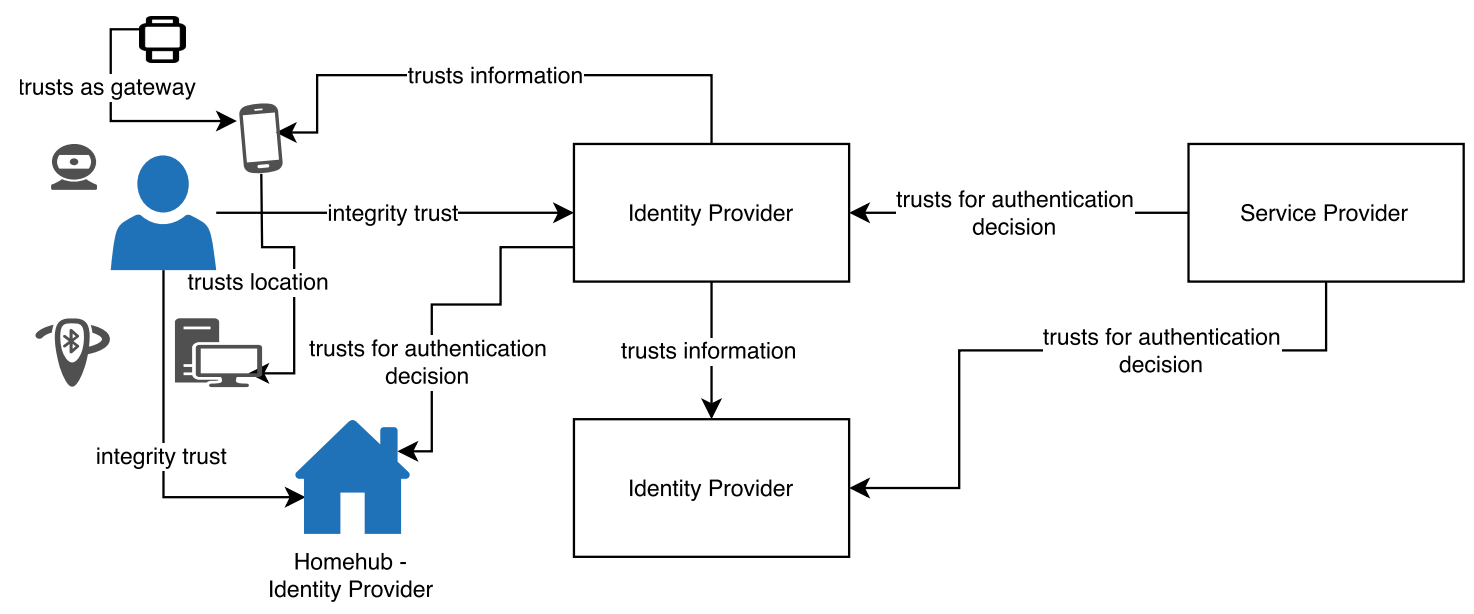

Figure 1: Trust relationships for local and remote authentication use cases

other distributed risk-adaptive applications.

The paper is structured as follows. We carry out a requirements and gap analysis on trust models for our motivating scenario on multi-modal authentication in section 2. Section 3 describes the design and implementation of our trust management solution. We evaluate the feasibility of modeling and reasoning upon distributed trust relationships in section 4 . We discuss and compare our work in section 6 with related work on multi-modal authentication and policy-based and reputationbased trust models. We conclude with some final thoughts and directions for further research in section 7 .

\section{Trustworthy continuous multi-modal authentication}

In this section, we describe the trends that have lead to our motivating scenario on multi-modal authentication and briefly highlight how trust plays a role. Later on, we will discuss which challenges are not addressed by existing solutions and the stateof-the-art, and which trust relationships must be considered to bridge this gap.

\subsection{Trends and trust challenges for multi-modal authentica- tion}

Figure 1 depicts our high-level motivating scenario that we will use to illustrate the authentication related trends and trust challenges.

\subsubsection{Federated Single Sign On}

In federated Single Sign On (SSO), an identity provider (IdP) is taxed with authenticating users on behalf of a service provider (SP). The SP needs to trust the IdP to do a secure job of authenticating the user. The other way around, the user trusts the IdP with his credentials. The IdP itself is part of a federation and trusts the authentication assertions from the other IdP members in the circle of trust of IdPs and SPs. The trust in an IdP that offers multi-factor authentication (MFA) - i.e. combining factors representing something (1) you know, (2) you own, and/or (3) you are - will be higher than in one that only provides login/password authentication.

\subsubsection{Risk-adaptive authentication}

Contemporary authentication systems tap into the context of a user or service interaction [7, 8, 9] to ascertain the risk of a security threat. For example, online payments executed in the home country of a user are generally perceived as more safe than the same payment executed abroad. Wiring a small amount of money is less risky than a high amount. Risk-adaptive authentication schemes will verify additional non-intrusive context factors to offer a frictionless authentication experience in low-risk situations. This implies that trust relationships are context-dependent.

\subsubsection{Multi-modal authentication with behavioral biometrics}

Behavioral biometrics add a $4^{\text {th }}$ authentication factor based on something you do to multi-factor authentication systems. Typical examples are mouse and keystroke dynamics [10, 11, 12, 13], websites browsed and network traffic [14], stylometry [7], ambient sound [15] and gait recognition [16, 17]. While such behaviometrics are user friendlier, they have higher false acceptance rates (FAR) or false rejection rates (FRR) compared to iris scans or fingerprint biometrics. That is why multiple features or decisions are fused in multi-modal authentication [18] to obtain acceptable equal error rates (EER). Consider the example of a smart assistant homehub in Figure 1, which uses built-in voice recognition and identification to continuously authenticate users. On top of that it assesses the presence of wearable devices that use gait recognition to identify their bearer. It trusts these wearables to tell if they are currently being worn by the user or not.

To provide an accurate joint assessment of the identity of the subject, the contribution of each behaviometric will depend on its accuracy. Chair and Varshney's optimal decision fusion rule [3] assumes a static probability of correct identification and false alarms for each individual behaviometric. Each of the $n$ behaviometrics makes a local binary decision $u_{i}=\{-1,+1\}$ depending on whether it is in favor of the hypothesis $H_{0}$ or $H_{1}$ that either rejects or confirms the claimed identity of the sub- 
ject. The optimal decision fusion rule states that:

$$
\begin{array}{ll}
f\left(u_{1}, \ldots, u_{n}\right)=+1 & \text { if } a_{0}+\sum_{i=1}^{n} a_{i} u_{i}>0 \\
f\left(u_{1}, \ldots, u_{n}\right)=-1 & \text { otherwise }
\end{array}
$$

where the weights $a_{i}$ are computed as follows:

$$
\begin{gathered}
a_{0}=\log \frac{P_{1}}{P_{0}} \\
a_{i}=\log \frac{1-P_{i}^{M}}{P_{i}^{F}} \quad \text { if } \quad u_{i}=+1 \\
a_{i}=\log \frac{1-P_{i}^{F}}{P_{i}^{M}} \quad \text { if } \quad u_{i}=-1
\end{gathered}
$$

where the a priori probabilities $P_{0}=P\left(H_{0}\right)$ and $P_{1}=P\left(H_{1}\right)=$ $1-P\left(H_{0}\right)$ are assumed to be known, along with $P_{i}^{M}$ and $P_{i}^{F}$ respectively representing the FRR and FAR of the $i^{\text {th }}$ behaviometric.

\subsubsection{Limitations of decision fusion schemes}

While this decision fusion rule is used in multi-modal authentication schemes, it makes assumptions that - when invalid - may jeopardize the trustworthiness of the authentication scheme:

- The a priori probabilities $P_{0}$ and $P_{1}$ are fixed

- The probabilities $P_{i}^{M}$ and $P_{i}^{F}$ are statistically independent and fixed

- The sensors monitor the behavior of the same user and are congruent in time

- No sensor is compromised, nor is the data spoofed by an attacker

Indeed, considering the above smart homehub scenario in Figure 1 , the local decisions of the voice identification and gait analysis behaviometrics may not have been taken at the same time. Also, the error rates of gait analysis heavily depend on the position on the body where the accelerometer is worn (e.g. smartwatch vs. smartphone). Even if sampling on multiple positions on the body can improve the equal error rate of gait analysis [19], the obtained behaviometrics will not be statistically independent. Last but not least, from a security point of view, one must consider the fact that certain behaviometrics can be captured in a public setting by malicious adversaries that can later use them to spoof the identify of a subject. From the standpoint of the smart homehub, the availability of the wearables is not guaranteed, nor whether they are worn or carried at the same time by the same person, and neither is the fact that each wearable is known and trusted.

\subsubsection{Bridging the gap with a trust model}

As the above concerns influence the security trustworthiness of multi-modal authentication schemes, we aim to address this problem by selecting and composing behaviometrics at runtime driven by a trust model and management framework that is able to:

1. Broker trust relationships for sensors and their behaviometrics

2. Compose behavior data and individual behavioral authentication decisions

3. Account for availability and temporal discrepancies between behaviometrics

4. Revoke trust relationships for disconnected or compromised sensors

\subsection{Analyzing the stakeholders in trust relationships}

This subsection elicits the trust relationships following from the motivating examples described above. We identified four main stakeholders in our authentication use case:

- Identity Provider (IdP): completes the authentication

- Service Provider (SP): application requesting subjects to be authenticated

- Subject: the end-user to be authenticated

- Devices: delivering behavior and/or additional context information

As illustrated in Figure 1, these stakeholders trust each other for certain aspects. The SP trusts the IdP is competent to make authentication decisions, while the end-user trusts the IdP to store its credentials securely and not to share them with third parties nor use them for purposes other than authentication. The IdP in turn trusts the end-user's devices to deliver accurate behavior information. One device can trust another to share context information or function as a gateway, which encompasses that the device is competent to transfer the information securely and that it will do this truthfully.

\section{A trust model for multi-modal authentication}

Trust relationships are only implicitly evaluated in a coarsegrained manner in contemporary state-of-practice authentication systems. By declaring trust relationships and contextual dependencies explicitly in a model or policy, we can reason about trust and whether these policies hold in a particular context. Beyond declared in policies, trust between two entities can be built up. Reputation based systems learn which entities can be trusted in a given context. Thus, trust and distrust are either declared or learned within a certain context. This contextual information is provided together with the identifying behavior information by the devices of the end-user. Thus, the reliability of context data (e.g. time, location, proximity, on body or not) is subject to a trust assessment as well. The practical evaluation of context trustworthiness is out of scope for this work, and has been addressed in our previous work [20]. 


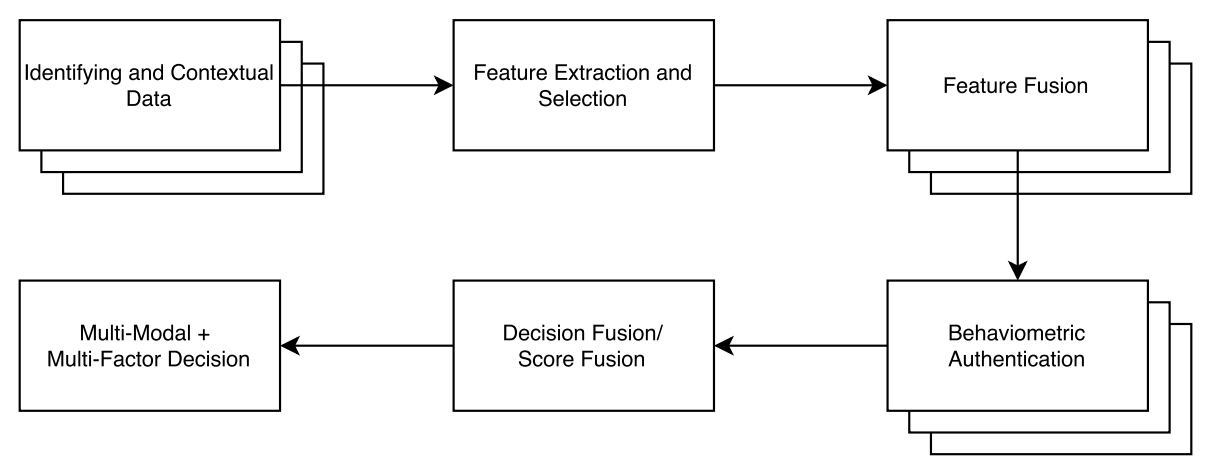

Figure 2: From behavior and context data to a joint multi-modal authentication decision

\subsection{High-level design and key trust relationships}

The whole authentication decision pipeline is shown in Figure 2. In principle, we consider trust as a transitive property. For example, the homehub may trust a known tablet (i.e. trust in a device) to deliver keystroke features, but not its accelerometer features (i.e. trust in the data) for gait authentication. When the homehub trusts a mobile device to deliver keystroke features as a behaviometric, and an application trusts the homehub as an IdP for authentication, then there is an implicit trust relationship between the application and the behaviometric. It also implies that if an IdP does not trust some behavior data it received from an end-user's device (i.e. trust in the data), it will no longer trust feature fusion schemes (i.e. trust in the data aggregation) and authentication modalities (i.e. trust in the individual decision) that depend on this data. When all authentication modalities end up being distrusted, the IdP may not be able to deliver a multi-modal authentication decision of a certain trust level to the SP.

Our trust model should support scenarios where the IdP and SP are remote online services (e.g. logging in to an online service with a Facebook account), local applications (e.g. the homehub identifying a household member to open and close doors), or combinations thereof (e.g. the homehub identifying a parent to place online orders). This means, however, that the selection of behaviometrics must be context dependent. For example, a user may be willing to share raw keystroke features to the homehub that implements keystroke dynamics as a behavioral authentication modality, but not to an online IdP due to the risk of leaking sensitive information. However, the online IdP may trust the individual behaviometric authentication decision of the homehub and fuse it with other authentication decisions for multi-modal authentication. This example clearly distinguishes the difference between trusting authentication features vs. trusting authentication decisions.

Based on the above use case, but also to support other scenarios, we succinctly list key requirements that must be fulfilled to establish trust for highly distributed risk-adaptive applications:

- R1. Application-agnostic trust model: The trust model must be able to express distributed trust relationships in an application independent way to increase interoperability.
- R2. Interpret and reason upon trust: The trust model must be grounded in a formal representation that supports reasoning upon trustworthiness such that each entity follows the same logic to infer and broker trust relationships.

- R3. Establish trust in different topologies: The trust model must have support to broker, revoke, fuse, and bootstrap trust relationships between trustors and trustees in a chain of trust (hierarchy), a circle of trust (fully connected network), and a web of trust (as in a social network)

- R4. Declare and learn trust relationships: The trust model must be able to manually express trust in a declarative way (e.g. using a policy) or learn the trustworthiness based on the reputation of trustees.

\subsection{Trust model for multi-modal authentication}

Our risk-adaptive trust model for authentication is shown in Figure 3. To fulfill the above requirements, we will elaborate on how we formalized our hybrid trust model into an ontology to benefit from the underlying description logic as a way (1) to guarantee a uniform interpretation of trust for all entities in a distributed application, and (2) to infer and broker trust relationships external to the authentication logic. The ontology can be extended with new concepts and instantiated with an editor like Protégé $5.2^{1}$ as depicted in Figure 4. The full model defines the classes and relationships between classes, and is available online $^{2}$. The most important formal relationships are discussed below.

Trust is a relation between two entities, the trustor and trustee. The trust relation implies that the trustor is willing to depend on the trustee for a certain commitment in a particular context. The trustor will assess the competence and integrity of the trustee. The concepts of competence and integrity were introduced in the computational trust model of Zhong et al. [21]. Competence captures the trustee's ability to perform a certain task in a specific situation, while integrity describes the belief that a trustee

\footnotetext{
${ }^{1}$ http://protege.stanford.edu/

${ }^{2}$ https://people.cs.kuleuven.be/ davy.preuveneers/ontologies/2017/07/trust.owl
} 


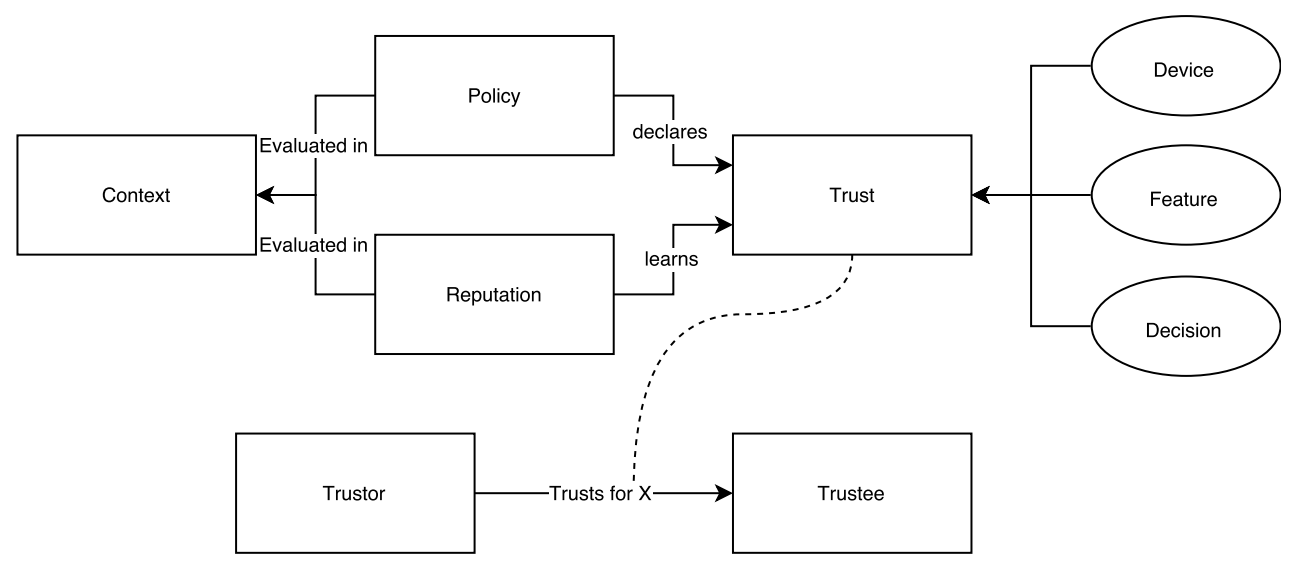

Figure 3: Risk-adaptive trust model for multi-modal authentication

is honest and acts in favor of the trustor. Competence tends to be relatively prone to changes, while integrity can change suddenly. One can estimate the competence and integrity based on previous actions and other information sources, e.g. when an authentication modality which is generally accepted to be good, consistently fails for a certain user, trust in this specific modality will diminish.

To infer trust relationships, we leverage description logic reasoning primitives to make implicit relationships explicit. We will illustrate this for our motivating use case with a few examples:

- tablet, homehub $\in$ Trustee: The tablet and homehub are trusted parties.

- homehub, idpl $\in$ Trustor: The homehub and idpl are trusting parties.

- Behaviometric $\sqsubseteq$ Factor: Declaring behaviometrics as a new subset of multi-modal authentication factors.

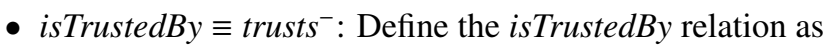
the inverse of the trusts relation.

- trusts $\sqcap$ distrusts $\equiv \emptyset$ : One cannot concurrently trust and distrust a party.

- trustsDevice, trustsFeature, trustsDecision $\sqsubseteq$ trusts : Model relations that respectively trust a device, feature and decision as subrelations of trusts.

- trustsCircle $\sqsubseteq$ trusts: By declaring it symmetric and transitive, the reasoner can infer implicit trust relationships between entities.

- implements(tablet, keystrokeTablet): The tablet device implements the keystroke dynamics authentication algorithm.

- implements(homehub, voiceHomehub): The homehub device implements the voice identification and authentication algorithm.

- trustsDevice(homehub, smartwatch): The homehub device trusts the smartwatch.
- trustsFeature(keystrokeHomehub, homehub): keystroke data is trusted to the homehub.

- trustsFeature(passwordIDP1, idpl): The idpl is trusted with password credentials.

- trustsDecision(idpl, homehub): The idpl trusts the homehub's decision.

- distrusts(keystrokeHomehub, idpl): The idpl is not trusted with keystroke data (e.g. for privacy reasons to not leak sensitive information).

- trustsCircle(idp1, gameService): The idp1 and gameService belong to a circle of trust.

\subsection{Reasoning upon complex trust relationships}

We declare trustsDecision as transitive properties, while not so for the trustsFeature property to prevent data leakage of privacy sensitive behaviometrics. With these and the above definitions, the reasoner can identify a chain of trust where the symmetric property does not hold. With these definitions, the reasoner can also infer that trustsCircle $\equiv$ trustsCircle ${ }^{-}$, or the inverse of the trustsCircle relationship is the same trustsCircle property. This means that if a Trustor trusts a Trustee, then the Trustee also trusts the Trustor. Also, as an entity can both be an instance of the Trustor and Trustee class, it can express trust in itself (making circleOfTrust a reflexive property) to characterize whether an entity has been compromised.

We will provide examples with growing complexity that demonstrate the added value of modeling trust relationships with description logics (DL). To do so, we will show the results of DL queries, and explain how the DL reasoner came to these conclusions. Protégé allows for specifying such queries in Manchester syntax [22] $]^{3}$ in the form of class expressions. A class expression defines a set of individuals by formally specifying conditions on the individuals' properties. A class expression can be simply the name of class (e.g. Trustee) or possibly the combination of such classes and class restrictions us-

\footnotetext{
${ }^{3}$ https://www.w3.org/TR/owl2-manchester-syntax/
} 


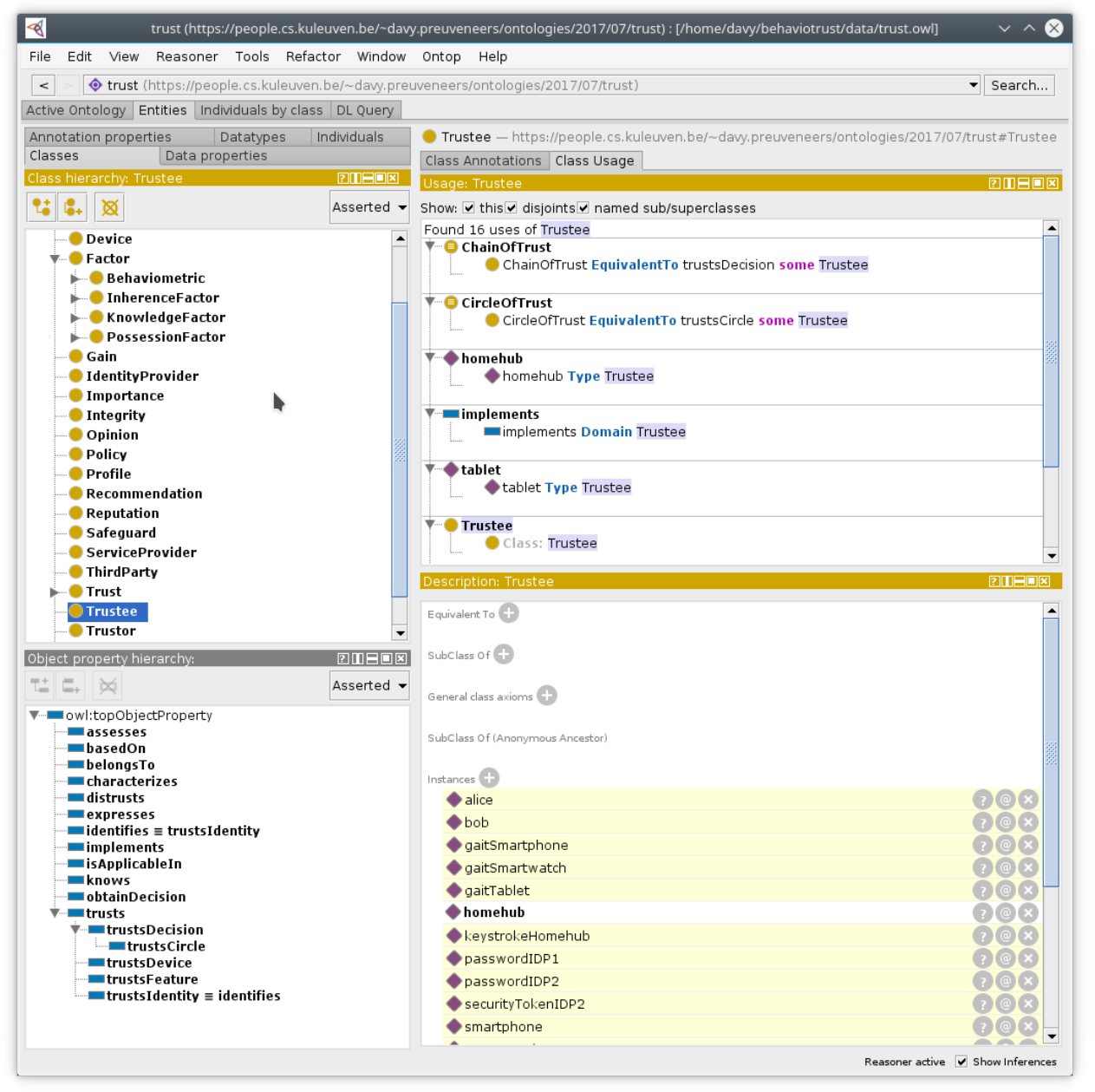

Figure 4: Formalization of the trust model into an ontology with Protégé

ing boolean connectives, universal and existential quantification, number restrictions, etc. Figure 5 depicts an example of such a class expression with the instance $i d p 2$ belonging to the given class expression.

The individual alice is identified as an instance of the class expression Trustor, even if we did not explicitly declare alice to be an instance of the class Trustor. By formally describing all relationships with description logic, the reasoner can infer this property by exploiting the following axioms:

1. alice trustsFeature gaitTablet: we declared in the ontology that alice trusts the gait recognition feature of the tablet.

2. trustsFeature SubPropertyOf: trusts: we declared trustsFeature to be a specialization of the trust relationship.

3. trusts Domain Trustor: we declared any individual with a trust relationship to be a member of the Trustor class.

The following class expression identifies those individuals that trust the decision of $i d p l$ :

\section{(trustsDecision value idpl)}

The next class expression matches individuals that trust the decision of any entity that implements a password factor:
(trustsDecision some (implements some PasswordFactor))

In the previous example, any instance of the class PasswordFactor will be implicitly checked. In our configuration, we declared two identity providers that each implemented password authentication, resulting in respectively two individuals passwordIDP1 and passwordIDP2.

- PasswordFactor: passwordIDP1, passwordIDP2

- (implements some PasswordFactor): idp1, idp2

- (trustsDecision some (implements some PasswordFactor)): idp1, idp2, gameService, mailService

For example, the last class expression states that mailService is a matching individual. The ontology reasoner inferred this based on the following axioms:

1. passwordIDP2 Type PasswordFactor

2. Symmetric: trustsCircle

3. trustsCircle SubPropertyOf: trustsDecision

4. idp2 trustsCircle mailService

5. idp2 implements passwordIDP2 


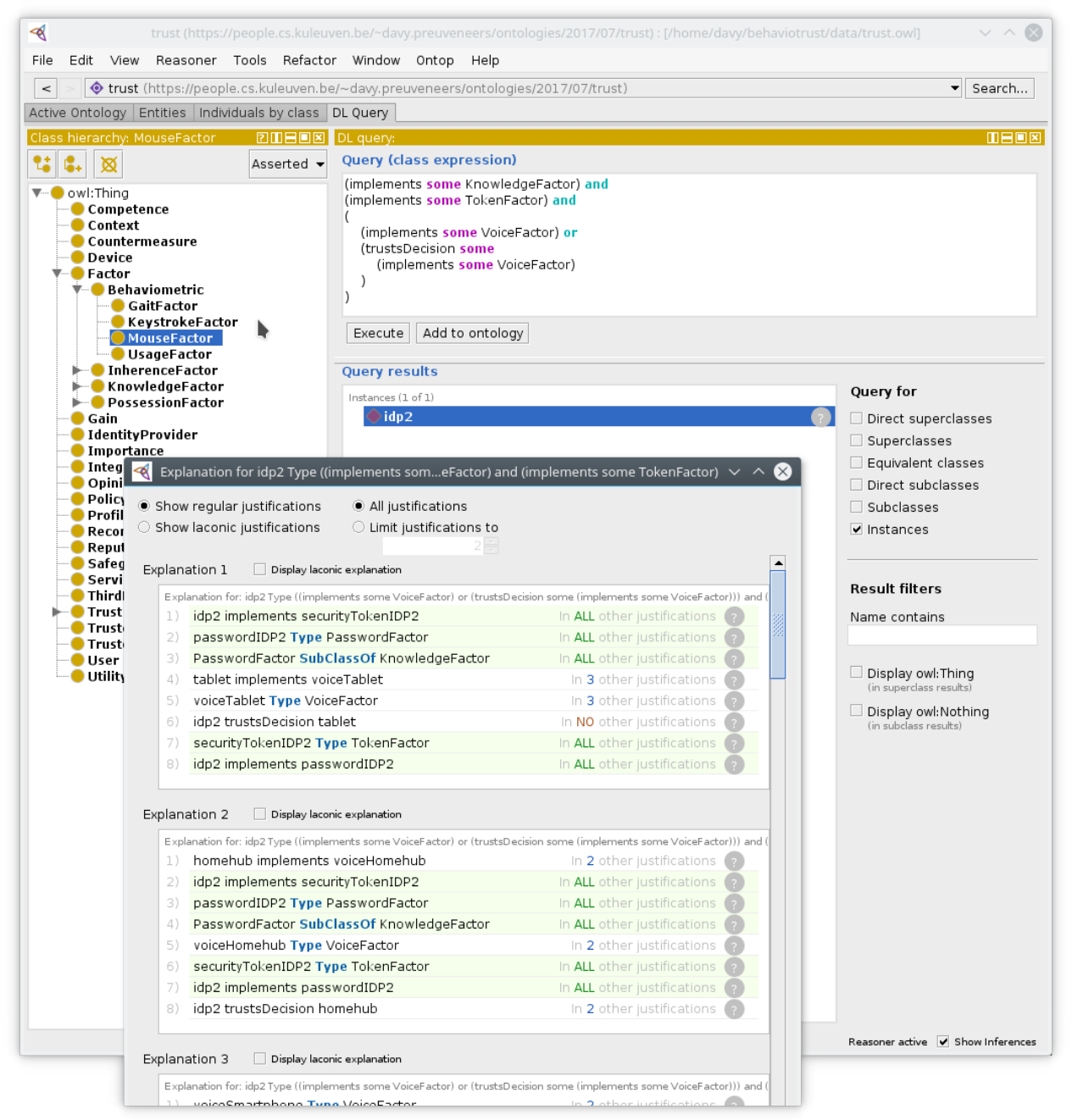

Figure 5: Reasoning upon and explaining complex trust relationships with Protégé

The following example is a more elaborate class expression that aims to establish which of the two services provides a multifactor authentication scheme for user alice. The class expression aims to find an identity provider whose decision is trusted:

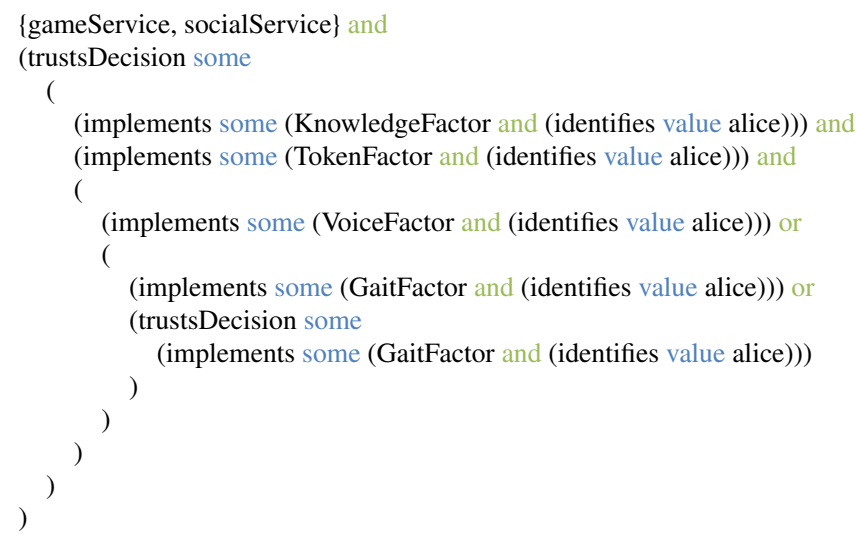

The identify provider must implement multi-factor authentication, combining a knowledge, possession and inherence factor. The latter can either be based on voice or gait. The identity provider must either implement the gait analysis itself, or rely on a third party that implements this authentication factor, and whose decision to authenticate alice is recursively trusted.

This multi-factor authentication example shows how our trust framework can reason upon implicit and explicit distributed trust relationships between users and identity providers, between identity providers and service providers in a circle of trust, data provided by sensors and devices, behaviometrics, etc. The outcome of the above class expression is gameService, and the explanation behind this outcome is based on logic inference on the following axioms:

1. idp2 implements securityTokenIDP2

2. alice trustsFeature gaitSmartphone

3. Symmetric: trustsCircle

4. alice trustsFeature passwordIDP2

5. PasswordFactor SubClassOf KnowledgeFactor

6. smartphone implements gaitSmartphone

7. gaitSmartphone Type GaitFactor

8. passwordIDP2 Type PasswordFactor

9. Transitive: trustsDecision 


\section{0. homehub trustsDecision smartphone}

11. alice trustsFeature securityTokenIDP2

12. trustsCircle SubPropertyOf: trustsDecision

13. securityTokenIDP2 Type TokenFactor

14. idp2 trustsCircle gameService

15. identifies InverseOf trustsFeature

16. idp2 implements passwordIDP2

17. idp2 trustsDecision homehub

The added value of our formal trust model is that the model can be further extended with domain specific concepts or properties without having to modify the underlying code. For example, the trustsDecision property can be further refined with subproperties to reflect reputation, competence or integrity characteristics of the Trustee (e.g. support for certificate based mutual authentication or other cryptographic capabilities).

In a similar fashion can our framework take contextual constraints into consideration to evaluate trust relationships. For example, when multiple devices are used to authenticate an individual (e.g. a smartwatch and a mobile phone), the above class expression can additionally state that these devices should all be at the same (semantic) location and/or that this location is a member of a particular trusted class (e.g. Work or Home). These contextual constraints and concepts are not modeled in the trust ontology, but in a separate context ontology [23] that is imported into the trust framework.

\subsection{Reputation and risk-adaptive trust relationships}

As previously stated, our trust model adopts a hybrid approach combining policy and reputation based knowledge representation techniques. The previous subsections focused on declarative policy models that formally represent various trust relationships in description logics. This subsection explains how the risk-adaptive trust model links reputation with the aforementioned description logic representations.

When multiple decisions must be combined in a multi-modal authentication flow, the decisions are initially fused according to Chair and Varshney's [3] optimal decision fusion rule. As explained in section 2.3.1, each factor is assigned a certain weight $w_{i}$ that corresponds with its accuracy, and as such reflects the reputation of the authentication factor. Authentication factors with a lower classification error rate will have a higher weight and a better reputation.

If the decision of authentication factor $i$ tallies with the decision of the fusion rule, then the weight $w_{i}$ is increased:

$$
w_{i} \leftarrow \min \left(1, w_{i} / \gamma\right) \quad \text { with } 0<\gamma<1
$$

If the decision of the authentication factor is in conflict, then its weight (and reputation) is decreased with a factor $\gamma$ :

$$
w_{i} \leftarrow w_{i} * \gamma
$$

To account for authentication factors that are not congruent in time, the weight $w_{i}$ is multiplied with an exponential factor so that the impact of an old value is less than that of a recent value:

$$
w_{i} \leftarrow w_{i} /\left(1+2^{t-s}\right)
$$

The parameter $s$ represents an acceptable time difference. As long as $t$ is much smaller than $s$, the weight $w_{i}$ is not significantly changed. For $t=s$, the weight $w_{i}$ is reduced to half its value. For larger values of $t$, the weight is reduced even further.

When the weight $w_{i}$ is below a certain threshold $\theta$, then it triggers a distrusts declaration in the trust ontology such that the authentication is no longer taken into consideration when establishing a trustworthy combination of authentication factors.

This adaptive reputation scheme addresses the limitation of the decision fusion scheme that assumes fixed a priori probabilities (see section 2.1.4), and it is also useful to detect inconsistencies between statistically dependent authentication factors. This way, our scheme can detect and eliminate both faulty and compromised authentication factors.

\subsection{Implementation}

Our Java-based trust management framework is built as a standalone Spring Boot ${ }^{4}$ 1.5.4 application with REST APIs, and integrates the Hermi ${ }^{5}$ 1.3.8 OWL ontology reasoner to infer trust relationships. When an application or service (i.e. the $S P$ instance of Trustor) requires a user to be authenticated, it aims to find a possible solution for a query where:

- The SP trusts an IdP

- The user trusts the same IdP

- The IdP meets the authentication factor requirements (number or type)

The IdP meets these requirements if it implements these authentication factors itself. This means it must be trusted with the data or features required for this factor, either directly or indirectly through a chain of trustees to securely forward the data. Otherwise, it must trust another Trustee instance that implements this authentication factor on the condition that that one is trusted with the right data. In a distributed setup, each node has deployed the Spring Boot based trust framework, and the nodes communicate with one another through the REST APIs to broker the trust relationships.

These REST APIs are also used to broker distrust relationships (see some examples in the above list). These are triggered automatically by our framework in the following cases:

1. There is no behaviometric data or the device/sensor is unavailable

2. The behaviometric data is too old

3. The behaviometric data is inconsistent

4. A required context constraint (time, location, proximity, onbody) is not valid

For example, consider a scenario where the accelerometer features of a smartwatch and a smartphone are both fused to elicit a single gait analysis authentication decision (i.e. feature fusion rather than decision fusion). If both sensors provide inconsistent data, then both feature data streams are discarded.

\footnotetext{
${ }^{4}$ https://projects.spring.io/spring-boot/

${ }^{5}$ http://www.hermit-reasoner.com/
} 


\begin{tabular}{|c|c|c|c|c|}
\hline \multicolumn{5}{|c|}{ (4 Item(s)) } \\
\hline \multirow{2}{*}{\begin{tabular}{|c|} 
Add \\
$\square$ 圆
\end{tabular}} & Remove $\mathrm{P}$ & Reorder & & \\
\hline & Instance & & Criteria & Options \\
\hline$\square$ & DataStore & 1 & REQUISITE ম & \\
\hline$\square$ & ScriptedMatch & 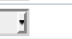 & SUFFICIENT & \\
\hline$\square$ & HOTP & 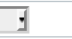 & REQUISITE ] & \\
\hline & ScriptedSave & 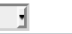 & REQUISITE d & \\
\hline
\end{tabular}

i This table lists the authentication modules that make up this authentication chain.

Figure 6: Chain of scripted step-up authentication with ForgeRock OpenAM.

Such inconsistencies are identified with signal processing and anomaly detection algorithms embedded in the framework, and not directly in the trust model. However, when the framework recognizes the behaviometric feature of a trustee is consistently inconsistent, it will trigger a distrust relationship declaration for this behaviometric feature that the ontology reasoner will then take into consideration. Whenever such a distrust relationship is declared or inferred, the corresponding authentication pipeline as depicted in Figure 2 is not trusted as a whole and will be discarded to authenticate the user.

The advantage of using a description logic reasoner like Hermi T is that it automatically handles the complexity of making implicit trust or distrust relationships explicit. This way, our trust framework can identify viable authentication chains that meet the authentication requirements of the application or service, even if at runtime there is a continuous evolution of trust and distrust relationships.

The current implementation relies on the HermiT reasoner to be embedded in each Spring Boot based trust framework instance with the trust ontology being part of its knowledge base. However, each application instantiates the ontology based on the capabilities being provided. A consequence is that distributed description logic reasoning is not possible. This design choice was deliberate as in our experimental setup, mobile devices can come and go, and subsequently also the behavioral authentication building blocks that they may offer.

Each trust framework instance can reason upon its own individuals and axioms, as well as those provided by other connected trust framework nodes in the network. The distributed nature of our trust framework does not rely on a centralized architecture, but rather on a distributed hash table (DHT) for decentralized peer-to-peer computer networks. The current implementation makes use of the Kademlia [24] DHT to have nodes exchange information with one another.

\section{Evaluation}

In this section, we will evaluate our trust model and supporting management framework from both a qualitative and quan- titative perspective, not only to assess whether the challenges in section 2 and the requirements identified in section 3 have been addressed, but also whether the deployment of our framework is practically feasible in terms of scalability and relative performance overhead.

\subsection{Experimental setup}

The experimental setup consists of a mobile device and a server side component designed around ForgeRock's OpenAM 14 platform as an identity provider, which offers scripting capabilities to easily plug in our trust-based multi-modal authentication functionality. Figure 6 illustrates the chain of authentication components to be executed when the user wants to log in:

1. DataStore: This LDAP component verifies the username and password credentials. This component is marked requisite which means that if this step fails, the whole authentication chain fails. Otherwise, we continue to the next component in the chain.

2. ScriptedMatch: This component calls our trust framework to analyze all trust relationships and check whether the behaviometrics were successful verifying the identity of the user. It is marked sufficient, which means if the trust-based multi-modal authentication passes, the authentication is successful. Upon failure, we go to step 3.

3. HOTP: This component will ask for the HMAC-based One Time Password sent by SMS or email. This component is marked requisite for the same reasons as the DataStore component.

4. ScriptedSave: When the HOTP component succeeds, the collected evidence will be stored for later comparison by the ScriptedMatch component.

\subsection{Qualitative evaluation}

Referring back to the goals set forward in section 2, we can conclude that our trust model and framework: 


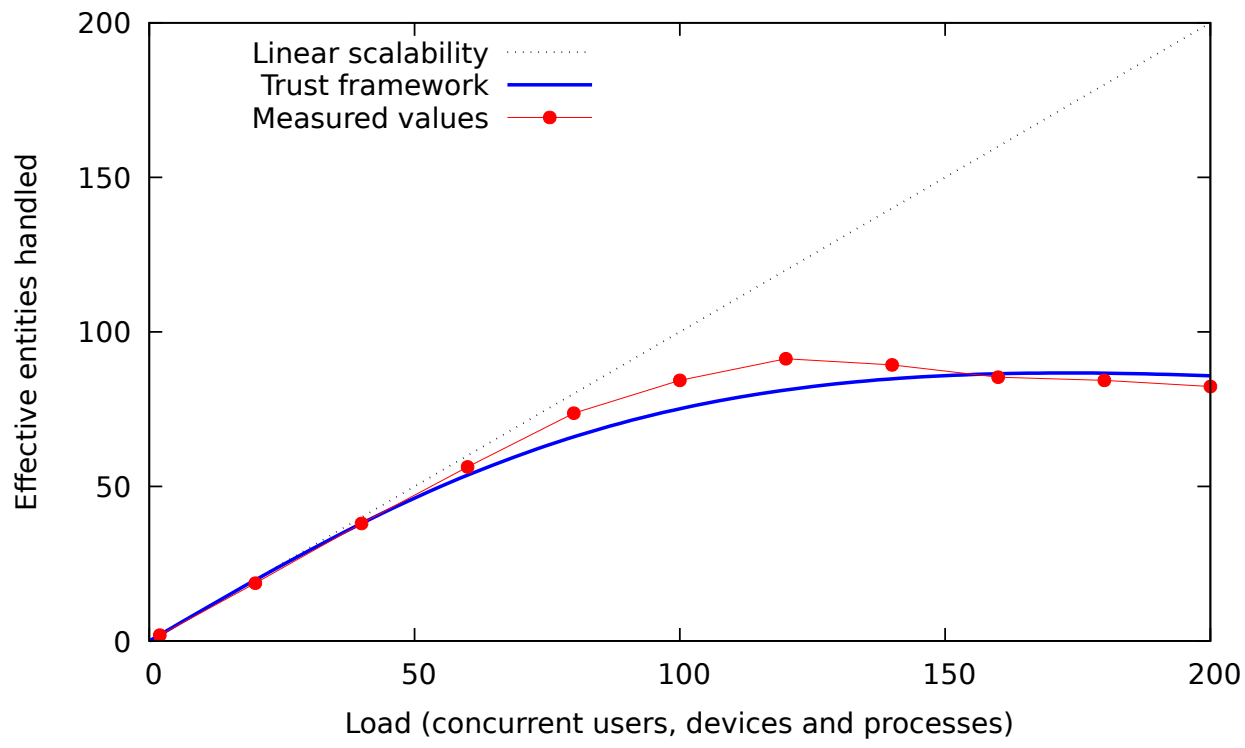

Figure 7: Performance and scalability evaluation on a Dell Latitude E6330 with 8GB of memory and an Intel Core i7-3520M CPU running at 2.90GHz.

- can infer and broker trust relationships between different kinds of trustors and trustees for various kinds of commitments

- can select and compose authentication pipelines that meet the requirements of the application, even in the presence of changing trust relationships

- accounts for the availability and temporal discrepancies between behaviometrics by automatically triggering distrust relationships

- allows to manually revoke trust relationships in case of compromised trustees (e.g. lost or stolen device)

By implementing the trust model as an ontology supported by a description logic reasoner, we fulfill the requirements ' $R I$ Application-agnostic trust model' and 'R2 Interpret and reason upon trust' identified in section 3 . The examples in the previous section provide evidence that requirements ' $R 3$ Establish trust in different topologies' and 'R4 Declare and learn trust relationships' have also been met.

\subsection{Quantitative evaluation}

To evaluate the practical feasibility of our trust model and framework, we assessed the performance relative to the number of users initiating authentication requests throughout the OpenAM platform. To do so, we simulated up to 200 entities (i.e. users and devices), and evaluated the impact. As the absolute performance numbers will be different when the trust framework is deployed on hardware with different computational resources, it is much more interesting to gain insights on the relative impact of how the trust framework behaves under growing workloads. By evaluating for 20, 40, 60 .. 200 concurrent entities, we can systematically evaluate the performance and scalability of the trust framework and create a model to predict how the system will behave under untested workloads. Preliminary experiments have shown for the current single node setup that the scalability is no longer near-linear for more than 100 entities. For more concurrent entities, one can deploy the trust framework on multiple nodes in a load-balanced setup to distribute the workload.

The underlying trust model was instantiated as follows:

1. Trust relations between SP and IdP

- Trustor $=$ SP

- Trustee = IdP

- Trust $=$ Password +2 out of 3 multi-modal authentication factors (gait, voice and keystrokes)

2. Trust relations between user and IdP

- Trustor $=$ User

- Trustee $=$ IdP

- Trust = Trust with user profile and password credentials

We simulated scenarios where every second one of the following security threats is triggered for $25 \%$ of the users:

- Password of user is leaked

- One of the behaviometrics is unavailable for 10 seconds

We carried out a systematic scalability assessment using the Universal Scalability Law [25, 26] (USL) demonstrating the performance feasibility of our trust framework. The USL combines (a) the initial linear scalability of a system under increasing load, (b) the cost of sharing resources, (c) the diminishing returns due to contention, and (d) the negative returns from incoherency into a model that defines the relative capacity $C(N)$ :

$$
C(N)=\frac{N}{1+\alpha(N-1)+\beta N(N-1)}
$$




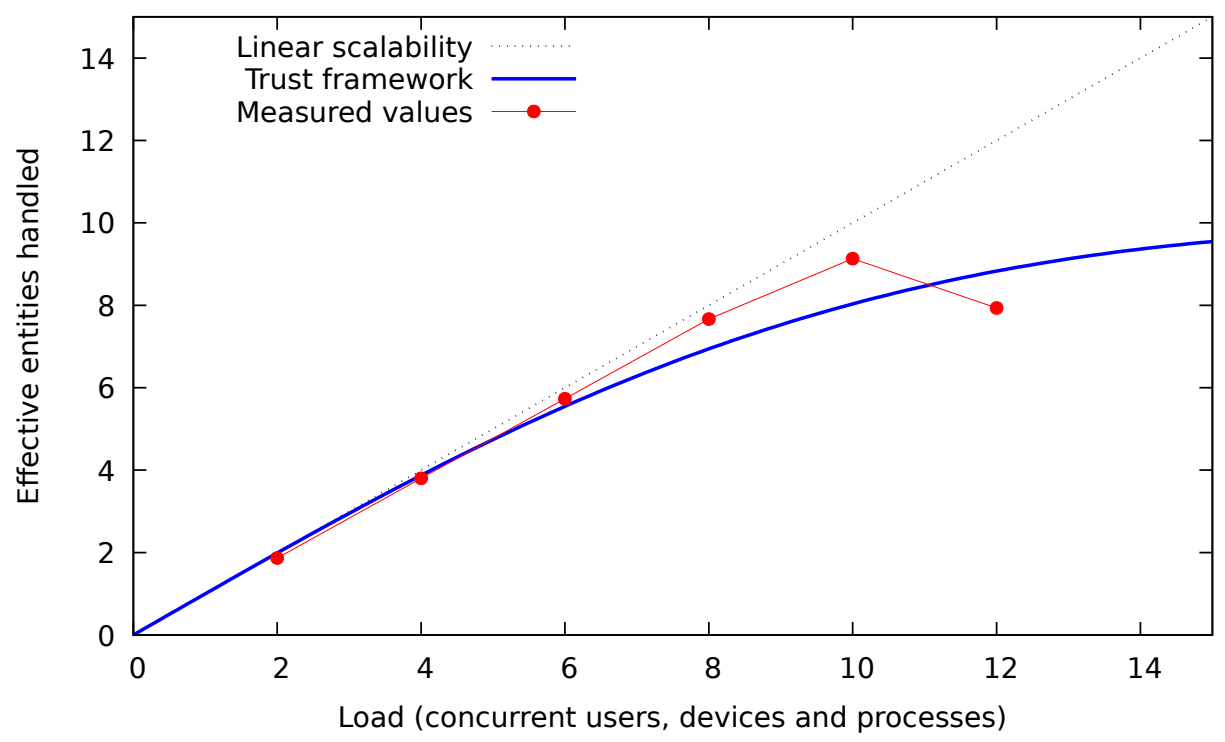

Figure 8: Performance and scalability evaluation on an ODRIOD-U3 mini-board with a 1.7GHz Quad-Core ARM processor and 2GB of RAM.

where $N$ represents the scalability of the software system in terms of the number of concurrent authentication requests of the different users, $\alpha$ represents the contention penalty, and $\beta$ defines the coherency penalty, with $0 \leq \alpha, \beta<1$. To benchmark the scalability, $N$ is incremented on a fixed configuration.

The results depicted in Figure 7 were obtained on a Dell Latitude E6330 with 8GB of memory and an Intel Core i7$3520 \mathrm{M}$ CPU running at $2.90 \mathrm{GHz}$. The graph shows that our trust framework scales sub-linearly up to about 90 users. We consider these results very acceptable given the fact that we simulated security breaches every second for $25 \%$ of the users. Last but not least, we compared the CPU time required by the OpenAM authentication platform and our trust framework, and noticed the CPU usage of our trust framework was about one third of the OpenAM platform, albeit the memory consumption was temporarily almost twice as high (about 0.9GB). While we did not further explored this in depth, we believe the higher memory consumption was due to the ontology reasoner caching intermediate results for improved performance.

Additional experiments of deploying the trust framework on an ODRIOD-U3 mini-board (see Figure 8 and 9) with a $1.7 \mathrm{GHz}$ Quad-Core ARM processor and 2GB of RAM showed acceptable performance for 10 users, with response times varying between 1 and 3 seconds under normal wireless network connectivity conditions. This demonstrates the feasibility of this combination for our homehub deployment. The assumption is that in the home environment, fewer users will need be authenticated concurrently. In fact, our experiments showed that the ARM processor was not the bottleneck, but rather the memory consumption of the trust framework. As the mini-board was running a full-fledged Ubuntu 16.04 Linux operating system with a graphical user interface, memory consumption could be improved by eliminating unnecessary components and services.

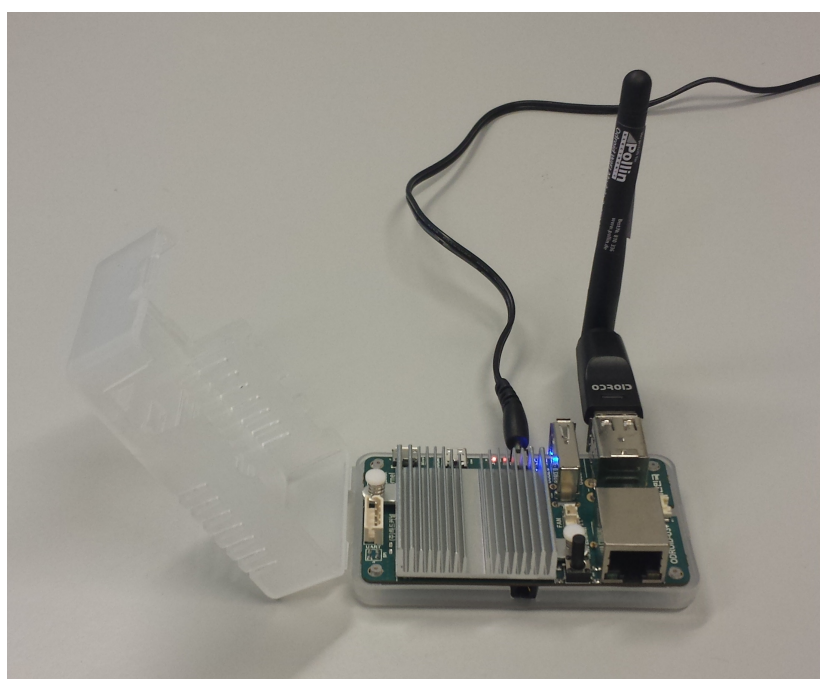

Figure 9: Evaluation on the ODRIOD-U3 mini-board.

\section{Validity threats, limitations and attack model}

In section 3 we formalized trust knowledge in description logic to allow distributed authentication systems to (1) externalize trust knowledge from application logic; (2) reason upon and broker trust relationships. This liberates application developers and identity providers from explicitly declaring all trust relationships themselves. Furthermore, by making trust an explicit concept, it can be used to trace unwanted relationships. This is impossible in state-of-the-art authentication system where trust is an implicit concept. In section 4 we show that the encountered cost in terms of overhead is more than acceptable. However, we have to consider some validity threats to the obtained results. 


\subsection{Authentication chain complexity}

The results of our performance and scalability assessment depend on the complexity of the multi-modal authentication chain being requested by the service provider (see section 3.3 for examples). The examples we used in our experiments are sufficiently realistic. However, the more complex the combinations of multi-modal authentication factors, the longer it takes the first time to find a match and evaluate an authentication request. If sufficient memory is available, intermediate logic inference results can be cached for further reuse. Otherwise, subsequent requests will require more time as well.

\subsection{Overhead for small and static authentication chains}

The proposed trust framework is both powerful and complex due to its underlying description logic foundation and formal reasoning capabilities. However, when only a few entities (offering multi-modal authentication factors) will take part in a simple and static authentication chain, the proposed trust framework will have limited added value as all the trust relationships can be made explicit in advance. Especially in static authentication chains where all entities are fixed, there is no need to distinguish between different types of trust relationships or topologies, or to infer implicit relationships. In such scenarios, the reasoning engine of the trust framework will cause a relatively high computational and memory consumption overhead.

\subsection{Availability, connectivity and denial of service}

A multi-factor and multi-modal authentication system aims to verify the claimed identity of a user. As described in section 2 , this is done by evaluating the context of the user and numerous authentication factors and modalities. These evaluations are done by different entities in a distributed manner. A service provider will be able to make a final decision regarding the claimed identity using its trusted entities. In our system, these entities exchange trust knowledge to infer which other entities are trusted.

However, our trust framework assumes that sufficient authentication factors are available and that each entity can communicate with one another. This may not be the case, and in such a scenario the multi-factor authentication will fail if no alternative authentication chain can be found. Furthermore, a malicious adversary may disrupt one or more entities making the authentication factors temporarily unavailable to its intended users.

\subsection{Insider threats and attacks}

Also, when one trusted entity is providing false knowledge used in the description logic, incorrect or even malicious statements can be introduced. Thus, in the above examples, our system does not protect against parties that start behaving maliciously after being compromised. Therefore, entities must declare or learn their own trust relationships with the utmost care.

To address this concern, existing trust relationships can be verified - and revoked if necessary - by relying on redundant independent authentication factors that must be consistent with one another. This consistency can be validated through formal reasoning in description logic. However, when such an inconsistency is found, identifying the actual misbehaving entity and inferring whether this inconsistency is of an accidental or malicious nature remains non-trivial. Furthermore, consistency checking offers no protection against a malicious adversary that can compromise a majority or even all the entities involved.

\section{Related work}

In this section we describe the state of the art in multi-modal behaviometrics and trust models.

\subsection{Multi-modal behaviometrics}

The shift towards behaviometrics as a fourth authentication factor promises frictionless authentication and increased security. Different authentication modalities have been proposed, e.g. keystroke dynamics, gait authentication [27], bioimpedence [28], battery level [29], etc. These differ in applicability, availability and security. There is no one size fits all modality like with the other authentication factors. To decrease equal error rates (EER's) different fusion schemes have been proposed.

In the field of continuous authentication, Bailey et al. [30] tried both feature fusion and decision fusion of keyboard, mouse and graphical user interface interactions. Both fusion techniques performed better than each of the separate modalities. Decision fusion yields the best results with a false acceptance rate (FAR) of $2.10 \%$ and a false rejection rate (FRR) of $2.24 \%$, at a detection speed of 10 minutes. Fridman et al. [1] perform decision fusion on three similar modalities: keystroke dynamics, mouse movement and stylometry. For each of these modalities they construct behaviometric based authenticators using a single feature as input. Decisions from 11 classifiers are fused using the optimal Chair and Varshney [3] rule as discussed in section 2.1. A FAR of $0.4 \%$ and a FRR of $1 \%$ is achieved, at a detection speed of 30 seconds. Saevanee et al. [31] did similar work for mobile devices. They perform decision level fusion on authentication modalities which use data from interactions with messaging apps. The fusion algorithm adopts weights equivalent to EERs of the individual modalities. Furthermore they propose a framework to incorporate intrusive authentication methods to support the continuous authentication. The achieved EER is $3.3 \%$. The reported detection speed is 2.3 minutes. The approach of Bailey et al., Fridman et al. and Saevanee et al. require that all modalities are always available and congruent in time. Furthermore they do not assess the trustworthiness of the data. This is certainly relevant since behavior can be observed, it is not a secret, thus it is more easily spoofed. On top of that, they lack adaptiveness. The context in which the identifying information is generated influences the individual authentication modalities, thus it needs to be taken into account. What is more, as discussed in section 2.1, FAR, FRR and EER are not fixed in time, and neither are the a priori probabilities.

A study by Dasgupta et al. [32] describes an adaptive selection technique for multi-modal multi-factor authentication. Their algorithm selects obtrusive and non-obtrusive authentication modalities and features for these modalities, based on 
contextual information. The context consists of the available modalities, the type of device (i.e. hand held, portable or fixed), the medium (i.e. wireless, wired or cellular), the surrounding conditions (i.e. background noise, lightening and surrounding motion) and the amount of user involvement required. The selection is done by solving a multi-objective non-linear quadratic optimization problem with probabilistic constraints. The model maximizes trustworthiness values, which are achieved by solving another optimization problem and exploiting the known EERs. Dasgupta et al. implemented the proposed selection scheme and proved its superiority over existing frameworks, by questionnaire. The reported FAR and FRR are respectively $3 \%$ and $4 \%$. The algorithm proposed by Dasgupta et al. can deal with unavailable modalities, but does not take the timeliness of a certain modality into account, e.g. the mouse dynamics data is one hour old. Furthermore there is no trustworthiness assessment of the provided identifying information. As in other work, the EER are assumed to be static. This selection technique could be used within our trust model. This would allow their system to take into account an even richer context.

\subsection{Trust models}

Trust management has been approached from two different perspectives: policy based trust and reputation based trust [6, 33]. The policy based approach tries to describe trust using predefined rules while relying on strong cryptographic assurances found in security certificates. Reputation based approach establishes trust based on direct experiences, which might be shared through a community.

\subsubsection{Policy based trust models}

Policy based trust frameworks allow to define what evidence should be presented by an entity, in what circumstances, in order to establish a trust relationship. Blaze et al. [34] first introduced the concept of "trust management". They defined it as: "a unified approach to specifying and interpreting security policies, credentials, and relationships which allow direct authorization of security-critical actions". "Security credentials" are "signed" attestations on aspects of the entity. Blaze et al. argued that the question answered by a trust compliance checker is not "is the key that signed this request authorized to take this action?", but is "does the set $C$ of credentials prove that the request $r$ complies with the local security policy $P$ ". Furthermore this question should not be answered by the application itself, but by a general trust compliance checker. In the following years several implementations of trust management frameworks as defined by Blaze et al. followed, such as Keynote [35, 36], REFEREE [37] and RT [38]. They describe different languages to define actions, policies and credentials. Our trust framework needs a similar language to express under what circumstances, certain entities are trusted. Not to make authorization decisions, but to successfully identify an entity.

\subsubsection{Reputation based trust models}

Different strategies to distribute trust in digital signatures necessary for credentials are well known, e.g. PKI [39] and
Kerberos [40]. However, trust in these keys can be learned [41]. A lot of effort has been spend to describe trust as a computational concept, using discrete [42, 43] or continuous [44, 45] values, or even expressing trust as a multidimensional value [41, 46, 47]. These metrics are used to encompass whether an entity is likely to engage in a trust relationship, in a certain context, for a certain aspect. Previous interactions is the input for most of these algorithms, which is why learning trust is often called reputation based trust. Recent work from Zhong et al. [21] distinguishes between competence and integrity trust. Competence trust captures the trustee's ability to perform a certain task in a specific situation. Integrity trust, on the other hand, describes the belief that a trustee is honest and acts in favor of the truster. This distinction is based upon studies in multiple domains [48]. The separation allows to use different statistical models to describe the trust values, i.e. they argue that the competence of a trustee will not change over time, while his integrity might be subject to fluctuations over time.

Learning trust is an important concept. In our trust model, trust can be learned using one of the schemes from literature. The distinction between competence and integrity trust is important for our authentication use case, as devices can be compromised or entities can be tempted leak identifying information.

\subsubsection{Hybrid trust models}

Hybrid trust frameworks combine the different properties of policy and reputation based trust frameworks. Policy based systems are intuitive and simple. They guarantee that nodes will comply with a certain behavior while relying on existing security mechanisms. Reputation based models allow to express trust based on behavioral evidence, making it no longer an intuitive concept, but something that can be evaluated in different contexts. Furthermore, such systems allow for dynamic adjustment of the trust relationships. One of the first attempts to integrate these perspectives [33] defined a trust management language allowing to specify policies based on numerical trust values. In [49] Lin et al. identify strengths and weaknesses of both models. They come up with a more general hybrid trust system, using the mobile agent system [50] as an example. Aivaloglou et al. [51] describe a hybrid trust management system for sensor networks. These types of networks typically have very strict computation and communication constraints. On top of that, nodes in these types of networks are heterogeneous, where each node typically has its own predefined role. It is this predeployment knowledge on the topology which is exploited by Aivaloglou et al. In order to achieve a flexible system they extend policies, based on the predeployment knowledge, with evidence from the information flows.

\section{Conclusion}

This paper presented a formal trust model for multi-modal authentication for distributed risk-adaptive applications. Evaluating trust mitigates the security risk introduced by fusing multiple authentication modalities. The model adopts a hybrid ap- 
proach, which combines policy and reputation based knowledge representation techniques. It externalizes trust knowledge from the authentication logic to achieve loosely coupled trust management, and formalizes this knowledge in description logic to infer and broker complex distributed trust relationships to make risk-adaptive decisions for multi-modal authentication. The formalization into an ontology and the use of description logic enables a uniform and consistent interpretation of risk and trust relationships. By externalizing trust knowledge from the application logic, our solution offers interoperability across information systems and applications with little to no impact on the application logic. Furthermore, the flexibility of our trust model enables stakeholders to further extend the trust model to introduce new concepts relevant within their application domain. The performance overhead appeared acceptable from a processing point of view, though the description logic reasoners consumed a reasonable amount of memory which may be a practical concern for resource constrained devices.

Future work will extend and evaluate our proof-of-concept in more realistic settings to test our distributed multi-modal authentication framework and measure the impact of network capacity constraints on the time required to reach consensus on which authentication pipeline to use path. Additionally, we will also evaluate other ontology reasoners to investigate whether lightweight alternatives, such as CEL offering OWL 2 EL (offering a subset of the description logic we currently use) can provide better support on resource constrained devices.

\section{Acknowledgments}

This research is partially funded by the Research Fund KU Leuven and DiskMan. DiskMan is a project realized in collaboration with imec. Project partners are Sony, IS4U and Televic Conference, with project support from VLAIO (Flanders Innovation and Entrepreneurship).

\section{References}

[1] L. Fridman, A. Stolerman, S. Acharya, P. Brennan, P. Juola, R. Greenstadt, M. Kam, Multi-modal decision fusion for continuous authentication, Comput. Electr. Eng. 41 (C) (2015) 142-156. doi:10.1016/j.compeleceng.2014.10.018.

URL http://dx.doi.org/10.1016/j . compeleceng. 2014.10.018

[2] P. K. Dhillon, S. Kalra, A lightweight biometrics based remote user authentication scheme for iot services, Journal of Information Security and Applications 34 (2017) 255 - 270. doi:http://dx.doi.org/10.1016/j.jisa.2017.01.003.

URL http://www.sciencedirect.com/science/article/pii/ S2214212616301442

[3] Z. Chair, P. K. Varshney, Optimal data fusion in multiple sensor detection systems, IEEE Transactions on Aerospace and Electronic Systems AES22 (1) (1986) 98-101. doi:10.1109/TAES.1986.310699.

[4] É. Dubois, P. Heymans, N. Mayer, R. Matulevičius, A Systematic Approach to Define the Domain of Information System Security Risk Management, Springer Berlin Heidelberg, Berlin, Heidelberg, 2010, pp. 289306. doi:10.1007/978-3-642-12544-7_16.

URL http: //dx.doi .org/10.1007/978-3-642-12544-7_16

[5] A. Jsang, R. Ismail, C. Boyd, A survey of trust and reputation systems for online service provision, Decision Support Systems 43 (2) (2007) 618 - 644, emerging Issues in Collaborative Commerce. doi:http://dx.doi.org/10.1016/j.dss.2005.05.019.
[6] D. Artz, Y. Gil, A survey of trust in computer science and the semantic web, Web Semantics: Science, Services and Agents on the World Wide Web 5 (2) (2007) 58-71.

[7] L. Fridman, S. Weber, R. Greenstadt, M. Kam, Active authentication on mobile devices via stylometry, application usage, web browsing, and GPS location, CoRR abs/1503.08479. URL http: //arxiv.org/abs/1503.08479

[8] F. Li, N. Clarke, M. Papadaki, P. Dowland, Active authentication for mobile devices utilising behaviour profiling, International Journal of Information Security 13 (3) (2014) 229-244. doi:10.1007/s10207-013-0209-6. URL http: //dx.doi .org/10.1007/s10207-013-0209-6

[9] E. Shi, Y. Niu, M. Jakobsson, R. Chow, Implicit authentication through learning user behavior, in: Proceedings of the 13th International Conference on Information Security, ISC'10, Springer-Verlag, Berlin, Heidelberg, 2011, pp. 99-113.

URL http://dl.acm.org/citation.cfm?id=1949317.1949329

[10] M. Antal, L. Z. Szabo, I. Laszlo, Keystroke dynamics on android platform, Procedia Technology 19 (2015) 820 - 826, 8th International Conference Interdisciplinarity in Engineering, INTER-ENG 2014, 9-10 October 2014, Tirgu Mures, Romania. doi:http://dx.doi.org/10.1016/j.protcy.2015.02.118.

URL http://www.sciencedirect.com/science/article/pii/ S221201731500119X

[11] Y. Deng, Y. Zhong, Keystroke dynamics user authentication based on gaussian mixture model and deep belief nets, ISRN Signal Processing 2013 (2013) 7. doi:10.1155/2013/565183.

URL https : //www . hindawi.com/journals/isrn/2013/565183/

[12] J. Wu, Z. Chen, An implicit identity authentication system considering changes of gesture based on keystroke behaviors, International Journal of Distributed Sensor Networks 11 (6). arXiv:http://dsn.sagepub.com/content/11/6/470274.full.pdf+html, doi: $10.1155 / 2015 / 470274$.

URL http://dsn. sagepub.com/content/11/6/470274.abstract

[13] P. S. Teh, A. B. J. Teoh, S. Yue, A survey of keystroke dynamics biometrics, The Scientific World Journal 2013 (2013) 24. doi:http://dx.doi.org/10.1155/2013/408280.

URL https://www . hindawi.com/journals/tswj/2013/408280/

[14] C. Imbert, Beyond the cookie: Using network traffic characteristics to enhance confidence in user identity (2014).

URL http://software-security.sans.org/resources/paper/ reading-room/cookie-network-traffic-characteristicsenhance-confidence-user-identity/

[15] N. Karapanos, C. Marforio, C. Soriente, S. Capkun, Sound-proof: Usable two-factor authentication based on ambient sound, in: 24th USENIX Security Symposium (USENIX Security 15), USENIX Association, Washington, D.C., 2015, pp. 483-498.

URL https: //www . usenix.org/conference/usenixsecurity15/ technical-sessions/presentation/karapanos

[16] A. Kale, N. Cuntoor, B. Yegnanarayana, A. N. Rajagopalan, R. Chellappa, Gait analysis for human identification, in: Proceedings of the 4th International Conference on Audio- and Video-based Biometric Person Authentication, AVBPA'03, Springer-Verlag, Berlin, Heidelberg, 2003, pp. 706-714.

URL http://dl.acm.org/citation. cfm?id=1762222.1762314

[17] C. Ntantogian, S. Malliaros, C. Xenakis, Gaithashing: A two-factor authentication scheme based on gait features, Computers \& Security 52 (2015) 17 - 32. doi:http://dx.doi.org/10.1016/j.cose.2015.03.009.

URL http://www.sciencedirect.com/science/article/pii/ S0167404815000413

[18] H. Saevanee, N. L. Clarke, S. M. Furnell, Multi-modal Behavioural Biometric Authentication for Mobile Devices, Springer Berlin Heidelberg, Berlin, Heidelberg, 2012, pp. 465-474. doi:10.1007/978-3-642-30436$1 \_38$.

URL http://dx.doi .org/10.1007/978-3-642-30436-1_38

[19] T. V. hamme, D. Preuveneers, W. Joosen, Improving resilience of behaviometric based continuous authentication with multiple accelerometers, in: G. Livraga, S. Zhu (Eds.), Data and Applications Security and Privacy XXXI - 31st Annual IFIP WG 11.3 Conference, DBSec 2017, Philadelphia, PA, USA, July 19-21, 2017, Proceedings, Vol. 10359 of Lecture Notes in Computer Science, Springer, 2017, pp. 473-485. doi:10.1007/978-3-319-61176-1_26. 
URL https : //doi.org/10.1007/978-3-319-61176-1_26

[20] J. R. Hoyos, J. Garca-Molina, J. A. Bota, D. Preuveneers, A model-driven approach for quality of context in pervasive systems, Comput. Electr. Eng. 55 (C) (2016) 39-58. doi:10.1016/j.compeleceng.2016.07.002. URL https://doi.org/10.1016/j.compeleceng.2016.07.002

[21] Y. Zhong, B. Bhargava, Y. Lu, P. Angin, A computational dynamic trust model for user authorization, IEEE Transactions on Dependable and Secure Computing 12 (1) (2015) 1-15. doi:10.1109/TDSC.2014.2309126.

[22] M. Horridge, P. F. Patel-Schneider, Owl 2 web ontology language: Manchester syntax, Tech. rep., W3C (2009).

URL http: //www . w3.org/TR/ow12-manchester-syntax/

[23] D. Preuveneers, J. Van den Bergh, D. Wagelaar, A. Georges, P. Rigole, T. Clerckx, Y. Berbers, K. Coninx, V. Jonckers, K. De Bosschere, Towards an Extensible Context Ontology for Ambient Intelligence, Springer Berlin Heidelberg, Berlin, Heidelberg, 2004, pp. 148-159. doi:10.1007/978-3-540-30473-9_15.

URL https : //doi .org/10.1007/978-3-540-30473-9_15

[24] P. Maymounkov, D. Mazières, Kademlia: A peer-to-peer information system based on the xor metric, in: Revised Papers from the First International Workshop on Peer-to-Peer Systems, IPTPS '01, Springer-Verlag, London, UK, UK, 2002, pp. 53-65.

URL http://dl . acm.org/citation. cfm?id=646334.687801

[25] N. J. Gunther, Guerrilla capacity planning - a tactical approach to planning for highly scalable applications and services., Springer, 2007.

[26] T. Heyman, D. Preuveneers, W. Joosen, Scalability analysis of the openam access control system with the universal scalability law, in: Future Internet of Things and Cloud (FiCloud), 2014 International Conference on, 2014, pp. 505-512. doi:10.1109/FiCloud.2014.89.

[27] H. Lu, J. Huang, T. Saha, L. Nachman, Unobtrusive gait verification for mobile phones, in: Proceedings of the 2014 ACM International Symposium on Wearable Computers, ISWC '14, ACM, New York, NY, USA, 2014, pp. 91-98. doi:10.1145/2634317.2642868.

URL http://doi .acm.org/10.1145/2634317.2642868

[28] I. Martinovic, K. Rasmussen, M. Roeschlin, G. Tsudik, Authentication using pulse-response biometrics, Commun. ACM 60 (2) (2017) 108-115. doi:10.1145/3023359.

URL http://doi .acm.org/10.1145/3023359

[29] J. Spooren, D. Preuveneers, W. Joosen, Leveraging battery usage from mobile devices for active authentication, Mobile Information Systems 2017 (2017) 14 URL https : //doi.org/10.1155/2017/1367064

[30] K. O. Bailey, J. S. Okolica, G. L. Peterson, User identification and authentication using multi-modal behavioral biometrics, Computers \& Security 43 (2014) 77-89.

[31] H. Saevanee, N. L. Clarke, S. Furnell, V. Biscione, Continuous user authentication using multi-modal biometrics, Computers \& Security 53 (2015) 234-246. doi:10.1016/j.cose.2015.06.001. URL http://dx.doi.org/10.1016/j.cose.2015.06.001

[32] D. Dasgupta, A. Roy, A. K. Nag, Toward the design of adaptive selection strategies for multi-factor authentication, Computers \& Security 63 (2016) 85-116. doi:10.1016/j.cose.2016.09.004.

URL http: //dx.doi.org/10.1016/j.cose.2016.09.004

[33] P. Bonatti, C. Duma, D. Olmedilla, N. Shahmehri, An integration of reputation-based and policy-based trust management, networks 2 (14) (2007) 10.

[34] M. Blaze, J. Feigenbaum, J. Lacy, Decentralized trust management, in: Security and Privacy, 1996. Proceedings., 1996 IEEE Symposium on, 1996, pp. 164-173. doi:10.1109/SECPRI.1996.502679.

[35] M. Blaze, J. Feigenbaum, A. D. Keromytis, Keynote: Trust management for public-key infrastructures, in: International Workshop on Security Protocols, Springer, 1998, pp. 59-63.

[36] M. Blaze, J. Ioannidis, A. D. Keromytis, Experience with the KeyNote Trust Management System: Applications and Future Directions, Springer Berlin Heidelberg, Berlin, Heidelberg, 2003, pp. 284-300. doi:10.1007/3540-44875-6_21.

URL http: //dx .doi .org/10.1007/3-540-44875-6_21

[37] Y.-H. Chu, J. Feigenbaum, B. LaMacchia, P. Resnick, M. Strauss, Referee: trust management for web applications, Computer Networks and ISDN Systems 29 (8) (1997) 953 - 964. doi:http://dx.doi.org/10.1016/S0169-7552(97)00009-3.

URL http://www.sciencedirect.com/science/article/pii/
S0169755297000093

[38] N. Li, J. C. Mitchell, W. H. Winsborough, Design of a rolebased trust-management framework, in: Security and Privacy, 2002. Proceedings. 2002 IEEE Symposium on, 2002, pp. 114-130. doi:10.1109/SECPRI.2002.1004366.

[39] S. Galperin, S. Santesson, M. Myers, A. Malpani, C. Adams, X. 509 internet public key infrastructure online certificate status protocol-ocsp.

[40] B. C. Neuman, T. Ts'o, Kerberos: an authentication service for computer networks, IEEE Communications Magazine 32 (9) (1994) 33-38. doi: $10.1109 / 35.312841$.

[41] A. JØsang, A subjective metric of authentication, Springer Berlin Heidelberg, Berlin, Heidelberg, 1998, pp. 329-344. doi:10.1007/BFb0055873. URL http: //dx.doi.org/10.1007/BFb0055873

[42] A. Abdul-Rahman, S. Hailes, Supporting trust in virtual communities, in: System Sciences, 2000. Proceedings of the 33rd Annual Hawaii International Conference on, 2000, pp. 9 pp. vol.1-. doi:10.1109/HICSS.2000.926814.

[43] A. Abdul-Rahman, S. Hailes, A distributed trust model, in: Proceedings of the 1997 Workshop on New Security Paradigms, NSPW '97, ACM, New York, NY, USA, 1997, pp. 48-60. doi:10.1145/283699.283739. URL http: //doi . acm . org/10.1145/283699.283739

[44] M. Stephen, Formalising trust as a computational concept, Ph. n dissertation. University of Stirling, scotland.

[45] R. Saadi, J.-M. Pierson, L. Brunie, T2d: A peer to peer trust management system based on disposition to trust, in: Proceedings of the 2010 ACM Symposium on Applied Computing, SAC '10, ACM, New York, NY, USA, 2010, pp. 1472-1478. doi:10.1145/1774088.1774403. URL http: //doi .acm.org/10.1145/1774088.1774403

[46] I. Ray, I. Ray, S. Chakraborty, An interoperable context sensitive model of trust, Journal of Intelligent Information Systems 32 (1) (2009) 75-104. doi:10.1007/s10844-007-0049-9. URL http://dx.doi.org/10.1007/s10844-007-0049-9

[47] B. Škorić, S. J. A. de Hoogh, N. Zannone, Flow-based reputation with uncertainty: evidence-based subjective logic, International Journal of Information Security 15 (4) (2016) 381-402. doi:10.1007/s10207-015-0298-5. URL http://dx.doi .org/10.1007/s10207-015-0298-5

[48] D. H. McKnight, V. Choudhury, C. Kacmar, Developing and validating trust measures for e-commerce: An integrative typology, Information Systems Research 13 (3) (2002) 334-359. arXiv:http://pubsonline.informs.org/doi/pdf/10.1287/isre.13.3.334.81, doi:10.1287/isre.13.3.334.81.

[49] C. Lin, V. Varadharajan, A hybrid trust model for enhancing security in distributed systems, in: Availability, Reliability and Security, 2007. ARES 2007. The Second International Conference on, 2007, pp. 35-42. doi:10.1109/ARES.2007.6.

[50] D. Chess, B. Grosof, C. Harrison, D. Levine, C. Parris, G. Tsudik, Itinerant agents for mobile computing, IEEE Personal Communications 2 (5) (1995) 34-49. doi:10.1109/98.468361.

[51] E. Aivaloglou, S. Gritzalis, Hybrid trust and reputation management for sensor networks, Wireless Networks 16 (5) (2010) 1493-1510. doi:10.1007/s11276-009-0216-8.

URL http://dx.doi .org/10.1007/s11276-009-0216-8 